\title{
A54 peptide-mediated functionalized gold nanocages for targeted delivery of DOX as a combinational photothermal-chemotherapy for liver cancer
}

\author{
This article was published in the following Dove Press journal: \\ International Journal of Nanomedicine \\ 20 July 2017 \\ Number of times this article has been viewed
}

Shengnan Huang, ${ }^{1} *$ Chunming $\mathrm{Li}^{2,}{ }^{2, *}$ Weiping Wang,' Huanjie Li,' Zhi Sun, ${ }^{3}$ Chengzhi Song, ${ }^{4}$ Benyi Li, ${ }^{5}$ Shaofeng Duan, ${ }^{6,7}$ Yurong $\mathrm{Hu}^{1,8,9}$

'Key Laboratory of Targeting Therapy and Diagnosis for Critical Diseases, School of Pharmaceutical Sciences, Zhengzhou University, Zhengzhou, People's Republic of China; ${ }^{2}$ Department of Pharmacy, Chongqing Cancer Institute \& Hospital \& Cancer

Center, Chongqing, People's Republic of China; ${ }^{3}$ Department of Pharmacy, the First Affiliated Hospital of Zhengzhou University, Zhengzhou, People's Republic of China; ${ }^{4}$ School of Physical Sciences, University of Science and Technology of China, Hefei, People's Republic of China; ${ }^{5}$ Department of Urology and Cancer Center, the University of Kansas Medical Center, Kansas City, KS, USA; ${ }^{6}$ College of Pharmacy, Henan University, Kaifeng, People's Republic of China; ${ }^{7}$ Department of Orthopedics, the First Affiliated Hospital of Zhengzhou University, Zhengzhou, People's Republic of China;

${ }^{8}$ Key Laboratory of Key Technology of Drug Preparation, Ministry of Education, Institute of Drug Discovery \& Development, Zhengzhou University, Zhengzhou, People's Republic of China; ${ }^{9}$ Collaborative Innovation Center of New Drug Research and Safety Evaluation, Zhengzhou, People's Republic of China

*These authors contributed equally to this work

Correspondence: Yurong Hu

Henan Key Laboratory of Targeting Therapy and Diagnosis for Critical Diseases, School of Pharmaceutical Sciences, Zhengzhou University, 100 Kexue Avenue, Zhengzhou City, Henan Province, 45000I, People's Republic of China

Tel +86 37| 65930224

Fax +86 37I 67739546

Email huyr@zzu.edu.cn

Shaofeng Duan

College of Pharmacy, Henan University, Jin Ming Avenue, Kaifeng City, Henan Province, 475004, People's Republic of China

Email sduan@henu.edu.cn
Abstract: The combination of photothermal therapy and chemotherapy (photothermalchemotherapy) is a promising strategy for cancer therapy. Gold nanocages (AuNCs), with hollow and porous structures and unique optical properties, have become a rising star in the field of drug delivery. Here, we designed a novel targeted drug delivery system based on functionalized AuNCs and evaluated their therapeutic effects in vitro and in vivo. We then loaded doxorubicin into this promising system, designated as DHTPAuNCs consisting of hyaluronic acid-grafted and A54 peptide-targeted PEGylated AuNCs. Its formation was corroborated by ultraviolet-visible spectroscopy, transmission electron microscopy and dynamic light scattering. This delivery platform needed hyaluronidase to release encapsulated drugs, meanwhile the acidic $\mathrm{pH}$ and near-infrared irradiation could accelerate the release. In addition, the results of cellular uptake demonstrate that this system could bind specifically with BEL-7402 cells. In vitro, we evaluated therapeutic effects of the DHTPAuNCs in BEL-7402 cells by 3-(4, 5-dimethylthiazol-2-yl)-2, 5-diphenyl-tetrazolium bromide assay. Moreover, in BEL-7402 tumor-bearing nude mice, its therapy effect in vivo was also evaluated. As expected, DHTPAuNCs exhibited excellent therapeutic effect by photothermal-chemotherapy, both in vitro and in vivo. In short, DHTPAuNCs with low toxicity showed great potential as a drug delivery system for cancer therapy.

Keywords: hepatocellular carcinoma, nanocarriers, targeted delivery, chemotherapy, photothermal therapy

\section{Introduction}

Hepatocellular carcinoma (HCC) is the second most common global cause of cancer-related deaths, and its increasing occurrence worldwide has been reported. ${ }^{1}$ Generally, the 5-year survival of patients with HCC is $<15 \% .^{2}$ Despite potentially curative treatments such as liver transplantation, radiofrequency ablation, and surgical excision, prospects for successful treatment remain poor. ${ }^{3}$ Thus, more efficient therapeutic approaches are still urgently needed.

Photothermal therapy (PTT), a minimally invasive local treatment, has attracted much attention in recent years because of its remote controllability, easy applicability, and low systemic toxicity and side effects. ${ }^{4,5}$ PTT utilizes heat generated from photothermal agents under near-infrared (NIR, $\lambda=700-1,100 \mathrm{~nm}$ ) light irradiation, ${ }^{6,7}$ where the absorption of tissues is quite limited, to cause irreversible cellular damage and subsequent tumor destruction because cancer cells are more heat-sensitive than normal ones. ${ }^{8}$ In PTT, photothermal agents are delivered to tumors either intravenously or 
intratumorally. The introduced photothermal agents can finally ablate the tumor through the raised localized temperature at the tumor site, which depends on absorbing and converting the penetrated NIR laser into heat. For efficient photothermal therapies for cancer treatment, researchers have widely explored various photothermal agents, including a variety of metallic nanostructures (such as Au-, Ag-, and $\mathrm{Cu}$-based nanoparticles), ${ }^{9-12}$ carbon nanomaterials (such as graphene and carbon nanotubes), ${ }^{13,14}$ transition metal chalcogenides, ${ }^{15}$ conjugated polymers, ${ }^{16}$ and small organic dyes. ${ }^{17}$ Among them, Au-based nanoparticles have been regarded as the most prominent candidates for biomedical applications owing to their merits such as precise control over their size and shape, facile surface functionalization, good biocompatibility, tunable localized surface plasmon resonance (LSPR), and high photothermal conversion efficiency. ${ }^{18}$ In addition to these properties, gold nanocages (AuNCs) bear a unique hollow and porous structure, ${ }^{19}$ and thus have become a new promising platform for biomedical applications correspondingly. However, there still remains a great challenge to realize their functions successfully in complex biological systems.

Hyaluronic acid (HA), a hydrophilic and linear negatively charged polysaccharide, is one of the extracellular matrix components and has been widely used as a superior biomaterial in some medical field as it is nonimmunogenic, ubiquitous, biocompatible, biodegradable and easily modified with other functional groups. $.^{20,21} \mathrm{High}-$ molecular weight (MW) HA can be specifically degraded by intracellular hyaluronidases (Hyals) to produce lower MW fragments and oligosaccharides, so that it has been widely applied in controlled-release drug delivery systems. ${ }^{22,23}$ Moreover, HA can bind with cluster determinant 44 receptor overexpressed in various malignant cancers and several other receptors on the surfaces of cells, including Toll-like receptors 2 and 4, receptor for hyaluronate-mediated motility, and HA receptor for endocytosis (HARE). ${ }^{24-26}$ Among them, HARE is expressed in the sinusoidal endothelial cells of lymph nodes, liver, and spleen. ${ }^{27}$ On the other hand, the half-life of intravenously administered HA is no more than 10 min because HA can be cleared rapidly in humans and a variety of animal species. ${ }^{21}$ However, a prerequisite of targeted delivery system is the long circulation period of nanoparticles in the blood, which is favorable for selective accumulation of nanoparticles in the tumor site. To conquer this limit, poly(ethylene glycol) (PEG) is commonly conjugated onto the nanoparticles. The PEG on the surface of the nanoparticles can improve the stability of the nanoparticles in blood and effectively reduce reticuloendothelial system uptake by preventing protein adsorption, which can increase their circulation time in blood. ${ }^{28}$

Higher specificity and better targeting efficiency can be achieved by modification of homing peptides. Homing peptides have been considered as the most promising targeting agents for human tumors owing to their several merits, including their relatively small molecular weights, ease of synthesis, low immunogenicity, good biocompatibility, and biodegradable characteristics in vivo. ${ }^{29}$ It has been reported that A54 peptide (sequence AGKGTPSLETTP) is the most specific and effective targeting agent to the BEL-7402 human hepatoma carcinoma cell line via cell surface markermediated endocytosis. ${ }^{30}$

In this work, we developed a novel tumor cell-specific targeted AuNCs-based drug delivery system (doxorubicin [DOX]-loaded, HA-grafted, and A54 peptide-targeted PEGylated AuNCs, abbreviated as DHTPAuNCs) and then investigated their therapeutic effects for the first time (Scheme 1). This system consists of a DOX-loaded AuNCs core surrounded by a HA shell, and functional groups on the surface of the DHTPAuNCs, including a hydrophilic polymer (PEG) and a promising targeting ligand (A54 peptide). The DHTPAuNCs can bind specifically with BEL-7402 cells, and then the coated HA polymer can be degraded in lysosomes, leading to an instantaneous release of encapsulated anticancer drugs into the tumor when activated by the Hyal in the presence of NIR laser and acidic $\mathrm{pH}$. In addition, the nanoparticles can selectively accumulate in the tumor site as the PEG prolonged their circulation time and A54 peptide enhanced their targeting ability. Our results demonstrated that DHTPAuNCs showed a synergistic effect in vitro and in vivo. It can be utilized as a powerful and promising photothermal-chemotherapy agent for treatment of HCC.

\section{Material and methods Materials}

$N$-hydroxysuccinimide (NHS, 98\%), ethylene glycol (99.5\%), 1-(3-dimethylaminopropyl)-3-ethylcarbodiimide hydrochloride $(\mathrm{EDC} \cdot \mathrm{HCl}, 98 \%)$, and sodium hydrosulfide $\left(\mathrm{NaHS} \cdot \mathrm{xH}_{2} \mathrm{O}\right)$ were all obtained from Aladdin (Shanghai, People's Republic of China). Poly (vinyl pyrrolidone) (PVP, average, MW $55,000)$, silver trifluoroacetate $\left(\mathrm{CF}_{3} \mathrm{COOAg}\right.$, more than $99.99 \%$ ), chloroauric acid $\left(\mathrm{HAuCl}_{4} \cdot 4 \mathrm{H}_{2} \mathrm{O}, 99.99 \%\right)$, propidium iodide, and 3-(4, 5-dimethylthiazol-2-yl)-2, 5-diphenyltetrazolium bromide (MTT) were purchased from Sigma Aldrich (Boston, MA, USA). Fetal bovine serum (FBS) and Roswell Park Memorial Institute medium (RPMI 1640) 
A
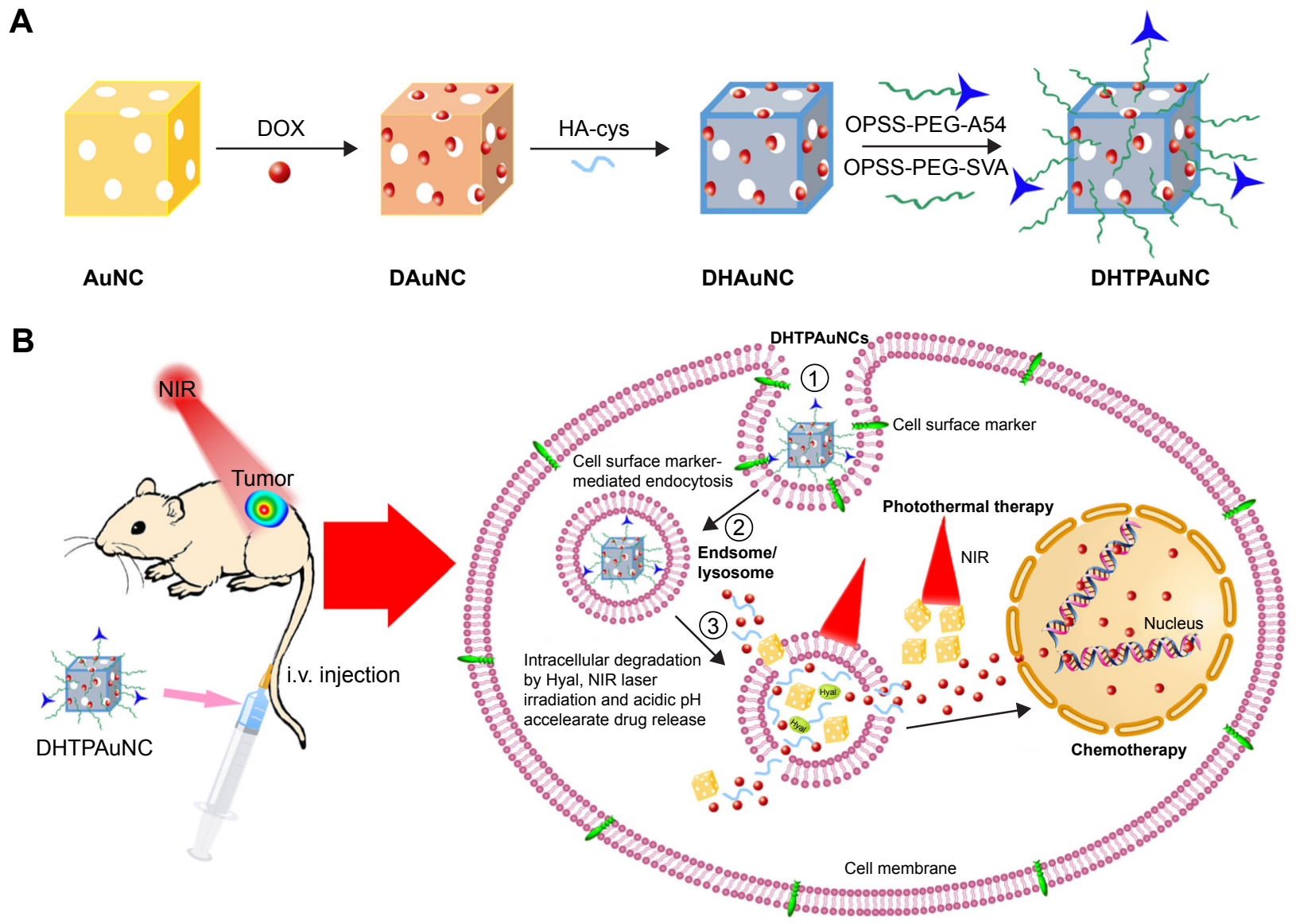

Scheme I Schematic representation for (A) the preparation of DHTPAuNCs and (B) hypothetical subcellular drug release behaviors and cellular uptake pathways. Abbreviations: AuNCs, gold nanocages; DAuNC, DOX-loaded AuNC; DHAuNC, DOX-loaded and HA-grafted AuNC; DHTPAuNCs, DOX-loaded, HA-grafted, and A54 peptide-targeted PEGylated AuNCs; DOX, doxorubicin; HA, hyaluronic acid; NIR, near-infrared irradiation.

were obtained from Biological Industries (Kibbutz Beit Haemek, Israel). Orthopyridyl disulfide polyethylene glycol succinimidyl valerate (OPSS-PEG-SVA, MW 2,000) was synthesized by Laysan Bio (Arab, AL, USA). Antimycotic solution and trypsin-ethylenediaminetetraacetic acid were purchased from Solarbio (Beijing, People's Republic of China). A54 peptide (sequence AGKGTPSLETTP) was obtained from ChinaPeptides Co., Ltd. (Shanghai, People's Republic of China). Throughout the experiment, ultrapure water (18.2 M $\Omega$; Millpore Co., USA) was used.

\section{Synthesis of AuNCs and HA-cysteine}

Synthesis of the AuNCs was performed as described in Xia's previous reports. ${ }^{31}$ In brief, Ag nanocubes (AgNCs) were synthesized using a rapid, sulfide-mediated polyol process with PVP serving as a capping agent. AuNCs were prepared through a galvanic replacement reaction between $\mathrm{HAuCl}_{4}$ and $\mathrm{AgNCs}$. When the LSPR peak at $760-800 \mathrm{~nm}$ was detected, we could confirm formation of the AuNCs. The resulting AuNCs were suspended in ultrapure water.
Synthesis of HA-cysteine (HA-cys): NHS (34.5 mg), HA (MW $100 \mathrm{kDa}, 100.0 \mathrm{mg}$ ), and 1-(3-dimethylaminopropyl)3-ethylcarbodiimide hydrochloride (EDC $\cdot \mathrm{HCl}, 53.9 \mathrm{mg}$ ) were dissolved in $50 \mathrm{~mL}$ of degassed phosphate-buffered saline (PBS) at the molar ratio of 1.2:1:1.2. After the addition of $15.2 \mathrm{mg}$ of cysteine, we stirred the mixture for $9 \mathrm{~h}$ with the $\mathrm{pH}$ value maintained from 4 to 6 . The purification process was realized by dialysis ( $\mathrm{MWCO}=14,000$ ) for $12 \mathrm{~h}$, repeating 4 times to remove unreacted cysteine against PBS ( $20 \mathrm{mM}, \mathrm{pH} 7.4$ ), followed by the storage in small vials under the protection of argon.

\section{Preparation of functionalized AuNCs}

To obtain DOX-loaded AuNCs (DAuNCs), the solution of DOX (0.2 mM) dissolved in PBS buffer (10 mM, pH 7.4) was mixed with AuNCs. After incubation for $12 \mathrm{~h}$ under stirring at room temperature, HA-cys was added to the mixture and the solution kept stirring for another $12 \mathrm{~h}$. Thereafter, the sample was centrifuged and washed three times with the aforementioned PBS until the superfluous DOX was 
removed, and thus we obtained DOX-loaded and HA-grafted AuNCs (DHAuNCs).

We conjugated A54 peptide onto the surface of DHAu NCs by a two-step protocol. First, A54 peptide (sequence AGKGTPSLETTP) and OPSS-PEG-SVA (MW 2,000) were dissolved in aqueous solution at a molar ratio of $1: 1.5$ at a total concentration of $0.12 \mathrm{mM}$ and stirred for $24 \mathrm{~h}$ at $4^{\circ} \mathrm{C}$ to generate OPSS-PEG-A54. The composition of OPSS-PEGA54 was characterized by ${ }^{1} \mathrm{H}-\mathrm{NMR}$. Second, OPSS-PEG (2.0 mg) or OPSS-PEG-A54 and OPSS-PEG (the molar ratio of OPSS-PEG and OPSS-PEG-A54 was 10:1 and the total weight was $2.0 \mathrm{mg}$ ) were added to the DHAuNCs solution or the HA-grafted AuNCs (HAuNCs) solution, and the mixture was stirred for $12 \mathrm{~h}$ at $4{ }^{\circ} \mathrm{C}$. Afterward, the solution was centrifuged and washed with PBS three times to remove the unattached OPSS-PEG-A54 or OPSS-PEG. The final products were separated by dialysis and lyophilized. Thus, we obtained the following functionalized AuNCs: DOX-loaded, HA-grafted and A54 peptide-targeted PEGylated AuNCs (DHTPAuNCs), HA-grafted and A54 peptide-targeted PEGylated AuNCs (HTPAuNCs), DOX-loaded and HAgrafted PEGylated AuNCs (DHPAuNCs), and HA-grafted PEGylated AuNCs (HPAuNCs).

\section{Physicochemical characterization measurement}

${ }^{1} \mathrm{H}-\mathrm{NMR}$ spectra were conducted on a Bruker DMX-500 spectrometer (Bruker, Germany). The optical properties were characterized using an ultraviolet-visible (UV-vis) spectrometer (Lambda 35, Perkine Elmer, USA). On a Malvern Nano ZS90 instrument (Malvern Instruments, UK), we determined the mean zeta potential and hydrodynamic particle diameter by laser Doppler velocimetry and dynamic light scattering. Transmission electron microscopy (TEM, FEI Tecnai 20, the Netherlands) was applied to analyze morphology of nanocomposites.

\section{Photothermal conversion of HTPAuNCs}

The experiment chose three concentrations of HTPAuNCs aqueous solution, namely, $10^{11}, 10^{10}$, and $10^{9}$ particles $\mathrm{mL}^{-1}$. The process was carried out as follows: $2 \mathrm{~mL}$ HTPAuNCs aqueous solution in a glass vial was irradiated by NIR laser (808 nm, $1.25 \mathrm{~W} \mathrm{~cm}^{-2}, 15 \mathrm{~min}$ ), and a digital thermometer was applied to measure and record the changes of temperature.

\section{Drug in vitro release}

To assess the DOX release from DHTPAuNC nanoparticles with NIR laser irradiation and Hyal at various $\mathrm{pH}$ values, we dispersed DHTPAuNCs in different buffer solutions with the presence or absence of Hyal. One milliliter of the DHTPAuNC sample containing about $60 \mu \mathrm{g}$ of DOX was exposed to the NIR laser ( $\left.808 \mathrm{~nm}, 1.25 \mathrm{~W} \mathrm{~cm}^{-2}, 10 \mathrm{~min}\right)$. Then, the samples were transferred to the dialysis bag ( $\mathrm{MWCO}=3,500 \mathrm{Da}$ ) and submerged into $20 \mathrm{~mL}$ of release medium ( $\mathrm{pH} 7.4$ PBS, $\mathrm{pH} 4.5$, and $\mathrm{pH} 5.5$ acetate buffer) with the presence or absence of Hyal $(150 \mathrm{U} / \mathrm{mL})$. The DHTPAuNC sample without NIR irradiation was used as a control. At each predetermined time point, the external release medium was replaced with an equal volume of fresh release medium, and the concentration of DOX was determined.

\section{Cellular uptake}

Human hepatoma carcinoma cell line BEL-7402 was purchased from Institute of Biochemistry and Cell Biology, CAS (Shanghai, People's Republic of China) and maintained in RPMI 1640 containing 10\% FBS and 1\% streptomycinpenicillin at $37^{\circ} \mathrm{C}$ in an atmosphere of $5 \% \mathrm{CO}_{2}$. To determine cellular uptake, cells were plated in triplicate at a density of $3.0 \times 10^{5}$ cells well ${ }^{-1}$ in six-well plates. After incubation for $24 \mathrm{~h}$, cells were washed with cold PBS three times, and treated with HPAuNCs or HTPAuNCs (AuNCs concentration at $0.625 \times 10^{10}$ particles $\mathrm{mL}^{-1}$ ) in $700 \mu \mathrm{L}$ serum-free fresh medium. After incubation for various times (1, 2, and $4 \mathrm{~h}$ ), the cells were rinsed with PBS three times and fixed for $15 \mathrm{~min}$ with 4\% paraformaldehyde. The cellular uptake was recorded by using a nanofluorescence microscopy (Olympus, Japan) (magnification: 400×). For quantification of HPAuNCs and HTPAuNCs in cells, after various time points $(0.5,1,2,4$, 6,8 , and $10 \mathrm{~h}$ ) incubation, Au content was measured with inductively coupled plasma mass spectrometry as described in Wang et al's previous reports. ${ }^{32}$

\section{Cell viability assays}

Bel-7402 cells were plated at a density of $1 \times 10^{4}$ cells well ${ }^{-1}$ in 96-well microplates. The cells were incubated with HTPAuNCs, free DOX, DHPAuNCs, or DHTPAuNCs with the same AuNCs and DOX concentration (AuNCs at the concentration of $0.625 \times 10^{10}, 1.25 \times 10^{10}, 2.5 \times 10^{10}$, and $5.0 \times 10^{10}$ particles $\mathrm{mL}^{-1}$, respectively, and the weight ratio of DOX/AuNCs was 1/16.3). The cells were exposed to PBS, HTPAuNCs, and DHTPAuNCs, and incubated at $37^{\circ} \mathrm{C}$ for $24 \mathrm{~h}$. Eight hundred eight nanometer laser $\left(1.25 \mathrm{~W} \mathrm{~cm}^{-2}\right.$, $10 \mathrm{~min}$ ) was used at 6-h incubation to achieve PTT. Cell viability was measured using the MTT assay according to the manufacturer-suggested procedures. ${ }^{33}$ 


\section{In vivo therapeutic evaluation of functionalized AuNCs with laser irradiation}

Female BALB/c nude mice (4-5 weeks old, 18-22 g) were purchased from Hunan SJA Laboratory Animal Co., Ltd (Hunan, People's Republic of China). All animal experiments complied with the National Laboratory Animal Management Regulations (People's Republic of China 1988) and Guidance on the Treatment of Experimental Animals (People's Republic of China 2006) and were conducted under a license with local ethical approval by the Life Science Ethics Committee Review Board of Zhengzhou University.

We used BEL-7402 tumor-bearing nude mice model to evaluate therapeutic effect of the functionalized AuNCs in vivo. This xenograft model was established by implanting $6 \times 10^{6}$ human BEL-7402 cells at right flank in female nude mice $(20 \pm 2 \mathrm{~g})$. The tumor volume was calculated as $0.5 \times$ (tumor length $) \times(\text { tumor width })^{2}$. When the tumor volume had developed to about $150 \mathrm{~mm}^{3}$, the mice were randomly divided into eight groups ( $\mathrm{n}=6$ per group): 1 ) normal saline (NS, control), 2) NS + NIR, 3) HTPAuNCs, 4) HTPAuNCs + NIR, 5) free DOX, 6) DHPAuNCs, 7) DHTPAuNCs, and 8) DHTPAuNCs + NIR. The dosage of DOX was normalized to be $2 \mathrm{mg} \mathrm{kg}^{-1}$ and the dosage of the carrier was normalized to be $32.6 \mathrm{mg} \mathrm{kg}^{-1}$, and at a frequency of every 3 days each tumor-bearing nude mouse was given an intravenous injection of $200 \mu \mathrm{L}$ the aforementioned solution for five times. For investigation of the PTT of the functionalized AuNCs in vivo, groups of (2), (4), and (8) were specifically irradiated for $10 \mathrm{~min}$ by a continuous NIR laser $(808 \mathrm{~nm}$, $1.25 \mathrm{~W} \mathrm{~cm}^{-2}$ ) at $4 \mathrm{~h}$ postinjection. Meanwhile, we applied an NIR thermal camera to monitor and record the tumor localized temperature changes under laser irradiation. Tumor growth was monitored during the following 15 days. The relative tumor volume RTV was applied as the index of antitumor effect, $\mathrm{RTV}=\mathrm{V}_{\mathrm{t}} / \mathrm{V}_{0}$, and $\mathrm{V}_{0}$ and $\mathrm{V}_{\mathrm{t}}$ were the tumor volumes before and after each treatment. After the final treatments, the mice were killed, and the tumors were harvested for histological examination. The tumors were collected and numbered for histopathological convention examination. The sections stained with hematoxylin-eosin were observed by an optical microscope (magnification: tumors samples $\times 200$ ).

\section{Serum biochemical assay}

To assay the effect of HTPAuNCs on kidney function and liver function, we carried out serum biochemical analysis using a standard protocol. Briefly, after the whole blood was centrifuged at 3,000 rpm for $15 \mathrm{~min}$, serum was collected from the supernatant, and then analyzed the serum by a biochemical autoanalyzer. Serum levels of tumor necrosis factor- $\alpha$ (TNF- $\alpha$ ), aspartate aminotransferase (AST), and alanine aminotransferase (ALT) were applied as indicators of liver function. And the indicators of kidney function were serum levels of urea (UREA) and creatinine (CREA).

\section{Statistical analysis}

Results are presented as mean \pm standard deviation (with a minimum of three independent experiments). Statistical differences between the groups were assessed using the unpaired Student's $t$-test. $P<0.05$ was considered to be statistically significant.

\section{Results}

\section{Preparation and characterization of AuNCs and functionalized AuNCs}

Our design mentioned above was realized by a series of experiments. First, AuNCs were prepared by a simple galvanic replacement reaction reported previously, and then characterized the AuNCs by TEM. As shown in Figure 1A, the size of AuNCs was about $45 \mathrm{~nm}$ and had a special cage structure with hollow interiors and porous (pore size $\sim 5 \mathrm{~nm}$ ), ultrathin walls. The obtained AuNCs showed good dispersibility and uniformity and its aqueous solution had a negative potential of $-12.84 \pm 1.25 \mathrm{mV}$. By changing the $\mathrm{Au} / \mathrm{Ag}$ alloy ratio, we prepared the AuNCs with an LSPR peak at about $770 \mathrm{~nm}$, which was conducive to PTT under laser irradiation (Figure 1D).

DOX applied in our research is a cytotoxic, anthracycline, topoisomerase II inhibitor and broad-spectrum antitumor drug. In the second step, DOX was loaded into the AuNCs $(-12.84 \pm 1.25 \mathrm{mV})$ to form DOX-loaded AuNCs (DAuNCs) nanoparticles $(-2.15 \pm 0.87 \mathrm{mV})$. In addition, the hydrodynamic diameter slightly increased from $98.23 \pm 1.25$ (AuNCs) to $99.34 \pm 1.91 \mathrm{~nm}$ (DAuNCs) after the formation of DOX-loaded AuNCs. In addition, UV-vis spectroscopy was employed to investigate the changes of optical properties before and after functionalizaion. The DAuNCs showed two characteristic absorption peaks: one in the visible region (about $490 \mathrm{~nm}$ ) from DOX and another in the NIR region (a broad plasma absorption band) from AuNCs (Figure 1D). The loading content of DOX (w/w\%) was $5.78 \%$.

In the third step, we synthesized and grafted HA-cys (HA $100 \mathrm{kDa}$ ) onto the surface of AuNCs to prevent the premature release of drugs. The unbounded HA was removed by continuous dialysis. The average number of HA molecules bound to a single AuNC was ca. 70, which could 
A

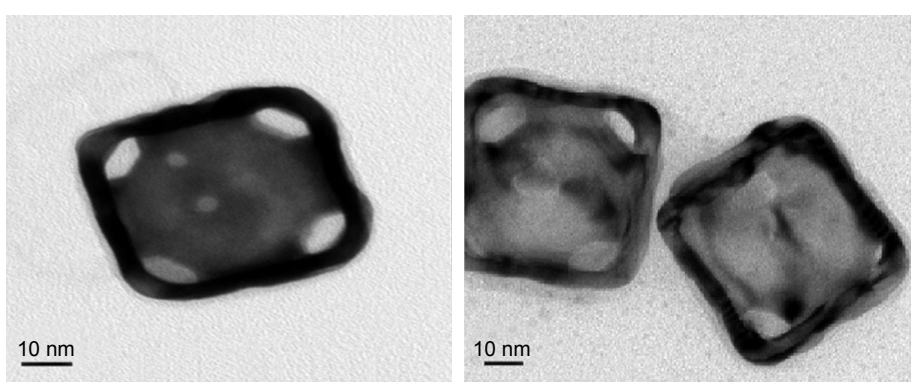

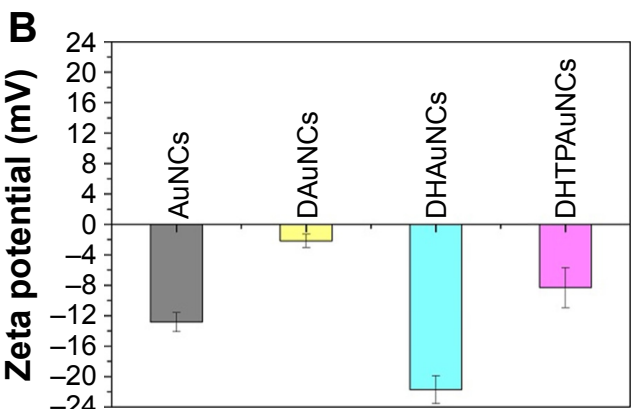

D

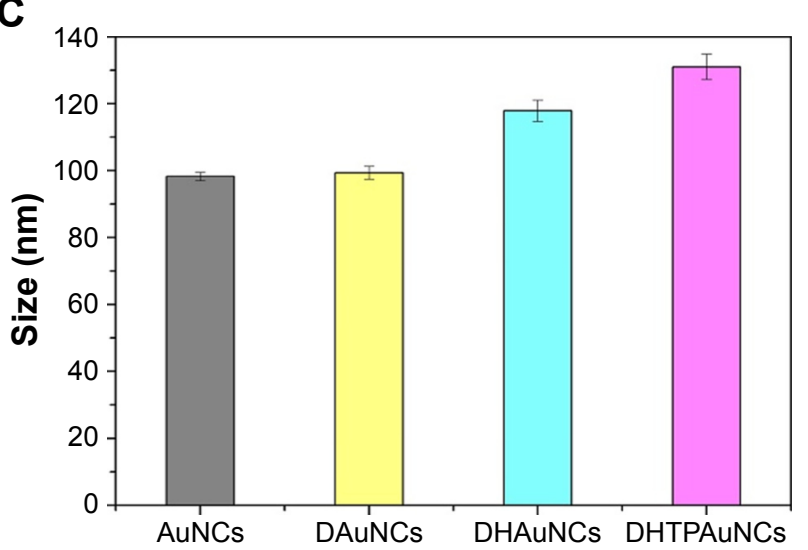

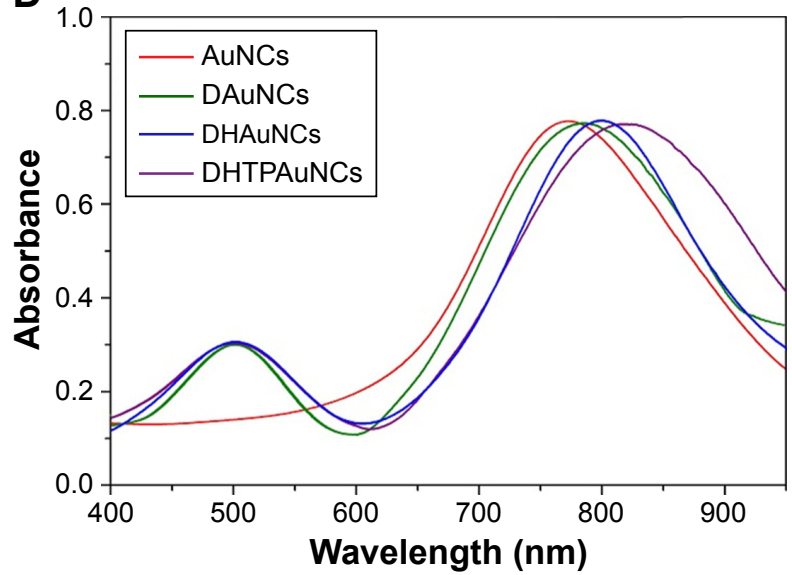

Figure I Characterization of the functionalized AuNCs.

Notes: (A) Transmission electron microscopy images of AuNCs (left) and DHTPAuNCs (right). (B) Zeta potentials of AuNCs, DAuNCs, DHAuNCs, and DHTPAuNCs. (C) Sizes of AuNCs, DAuNCs, DHAuNCs, and DHTPAuNCs. (D) Ultraviolet-visible spectra of AuNCs, DAuNCs, DHAuNCs, and DHTPAuNCs.

Abbreviations: AuNCs, gold nanocages; DAuNCs, DOX-loaded AuNCs; DHAuNCs, DOX-loaded and HA-grafted AuNCs; DHTPAuNCs, DOX-loaded, HA-grafted, and A54 peptide-targeted PEGylated AuNCs; DOX, doxorubicin.

be determined by hexadecyltrimethyl ammonium bromide turbidimetric method as reported before. ${ }^{34}$ The conjugation of HA dramatically reduced the surface potential to $-21.71 \pm 1.83 \mathrm{mV}$ and increased the viscosity and stability of the particles. In addition, the hydrodynamic diameter changed to $117.87 \pm 3.17 \mathrm{~nm}$ after the conjugation of HA onto DAuNCs, which was confirmed from the red shift in the LSPR band. The LSPR peak of DAuNCs appeared at $773 \mathrm{~nm}$ and that of DOX-loaded and HA-grafted AuNCs (DHAuNCs) shifted to $798 \mathrm{~nm}$ (Figure 1D).

As for application of AuNCs in vivo, it is critical to make the systemically administered nanoparticles to remain in blood circulation for a long period to achieve an effective concentration throughout tumors. Thus, the last step was the preparation of functionalized AuNCs by means of surface modification with PEG derivatives and liver cancer cellspecific ligand (A54 peptide) for active tumor targeting to enhance their preferable accumulation. PEG has been widely used for an improved blood circulation to increase the chance of their accumulation in tumors. First, OPSS-PEG-A54 was synthesized by the formation of amide bond between $-\mathrm{NH}_{2}$ group of A54 peptide and-COOH group of OPSS-PEG-SVA and characterized by ${ }^{1} \mathrm{H}-\mathrm{NMR}$ (Figure 2).

As seen from the top figure in Figure 2, the typical signal of $-\left(\mathrm{CH}_{2} \mathrm{CH}_{2} \mathrm{O}\right)_{\mathrm{n}}$ - on OPSS-PEG-SVA can be observed at $\delta 3.58 \mathrm{ppm}$. The peaks at $\delta 7.25,7.75$, and $8.30 \mathrm{ppm}$ correspond to the protons at the pyridine ring. The peak at $\delta 2.80 \mathrm{ppm}$ is from the two $-\mathrm{CH}_{2}-$ on the succinimide part. The $-\mathrm{CH}_{3}$ - of A54 can be observed at $\delta 0.75-0.81 \mathrm{ppm}$ (middle figure). From the ${ }^{1} \mathrm{H}-\mathrm{NMR}$ data of OPSS-PEGA54 (the bottom figure), we can see the typical signals of $-\left(\mathrm{CH}_{2} \mathrm{CH}_{2} \mathrm{O}\right)_{\mathrm{n}}$ - and the pyridine ring on OPSS-PEG-SVA at $\delta 3.58 \mathrm{ppm}, \delta 7.25,7.75$, and $8.30 \mathrm{ppm}$ and A54 peptide at $\delta 0.75-0.81 \mathrm{ppm}$. The peak at $\delta 2.80 \mathrm{ppm}$ was missing, indicating that the succinimide part on OPSS-PEG-SVA was replaced by A54 peptide. All these results demonstrated that the OPSS-PEG-A54 was successfully obtained. Subsequently, the mixture of OPSS-PEG-SVA and OPSS-PEG-A54 (the molar ratio of OPSS-PEG and OPSS-PEG-A54 was 10:1) was grafted onto the surface of DHAuNCs through the thioldisulfide exchange between the thiol group of cysteine conjugated with HA on the surface of DHAuNCs and the disulfide 

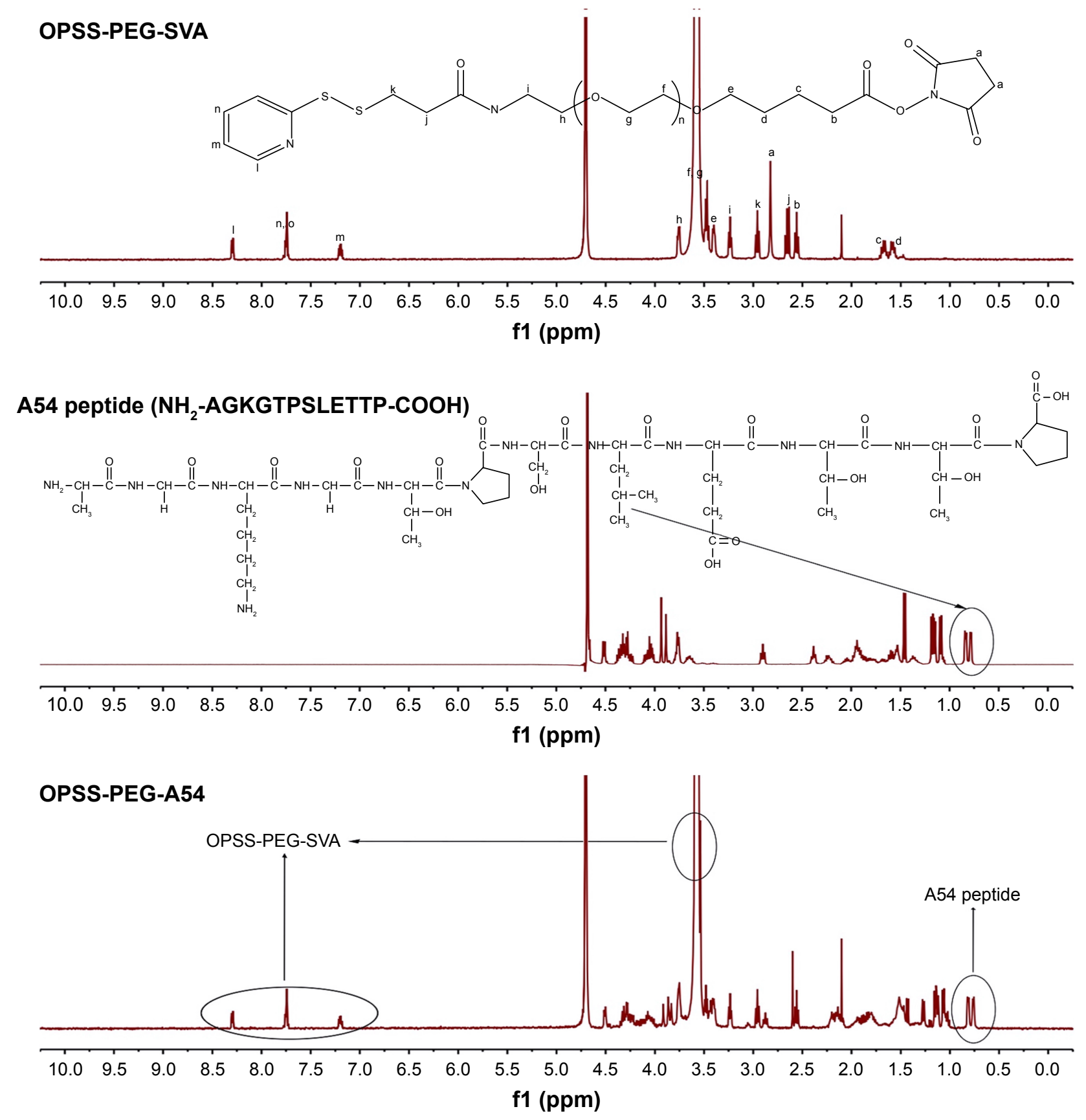

Figure 2 ' $H$-NMR spectra of OPSS-PEG-SVA, A54 peptide, and OPSS-PEG-A54 in $\mathrm{D}_{2} \mathrm{O}$.

Abbreviations: OPSS, orthopyridyl disulfide; PEG, poly(ethylene glycol); SVA, succinimidyl valerate.

of PEG derivatives. The release of 2-pyridinethione could be used to monitor the course of the reaction at $343 \mathrm{~nm} .{ }^{35}$ There were $\sim 800$ PEG chains on each AuNC. Compared with DHAuNCs, the LSPR peak of DOX-loaded, HA-grafted, A54 peptide-targeted PEGylated AuNCs (DHTPAuNCs) red shifted to $810 \mathrm{~nm}$, indicating the successful conjugation. The changes of hydrodynamic diameter $(131.01 \pm 3.78 \mathrm{~nm})$ and surface potential $(-14.32 \pm 2.64 \mathrm{mV})$ further confirmed the formation of DHTPAuNCs (Figure 1B and C).

\section{Photothermal conversion and the release profile of DHTPAuNCs}

To evaluate the potential application of DHTPAuNCs as a photothermal conversion agent for cancer therapy, DHTPAuNCs aqueous suspensions at various concentrations $\left(10^{9}, 10^{10}\right.$, and $10^{11}$ particles $\left.\mathrm{mL}^{-1}\right)$ and ultrapure water (control sample) were exposed to NIR laser irradiation. During this experiment, a thermal camera was used to measure and image the temperature changes. The laser heating 
curves of DHTPAuNCs were recorded (Figure 3A), demonstrating that the temperature of DHTPAuNCs aqueous solution could be raised from $25^{\circ} \mathrm{C}$ to $49.5^{\circ} \mathrm{C}\left(10^{9}\right.$ particles $\left.\mathrm{mL}^{-1}\right)$, $57.0^{\circ} \mathrm{C}\left(10^{10}\right.$ particles $\left.\mathrm{mL}^{-1}\right)$, and $62^{\circ} \mathrm{C}\left(10^{11}\right.$ particles $\left.\mathrm{mL}^{-1}\right)$ after NIR irradiation (Figure 3B).

In comparison, the temperature of the ultrapure water only increased by $6.5^{\circ} \mathrm{C}$. The strong LSPR of the DHTPAuNCs in the NIR region led to excellent photothermal properties, which was beneficial for the following PTT effect in vitro and in vivo. Moreover, the elevated temperature may accelerate the release of the encapsulated drugs. In addition, HA polymer grafted on the surface of DHTPAuNCs could be degraded in the absence of the intracellular enzyme Hyal, which could also be advantageous for drug release. First, we investigated the changes of hydrodynamic diameter and zeta potential of DHTPAuNCs and unmodified AuNCs in the presence of Hyal in $\mathrm{pH} 4.5$ acetate buffer. We found that the hydrodynamic diameter of DHTPAuNCs apparently decreased from $131.01 \pm 3.78$ to $101.23 \pm 1.82 \mathrm{~nm}$, which was close to that of unmodified AuNCs, and the zeta potential of DHTPAuNCs decreased slightly from $-8.32 \pm 2.64$ to $-7.23 \pm 2.82 \mathrm{mV}$; however, no significant changes were found in the hydrodynamic diameter and zeta potential of unmodified AuNCs. We ascribed this to the degradation of the HA on the surfaces of DHTPAuNCs in the presence of Hyal. Subsequently, we conducted a series of experiments under various conditions at the predesignated time points to further evaluate whether acidic $\mathrm{pH}$ and NIR laser irradiation

\section{A}
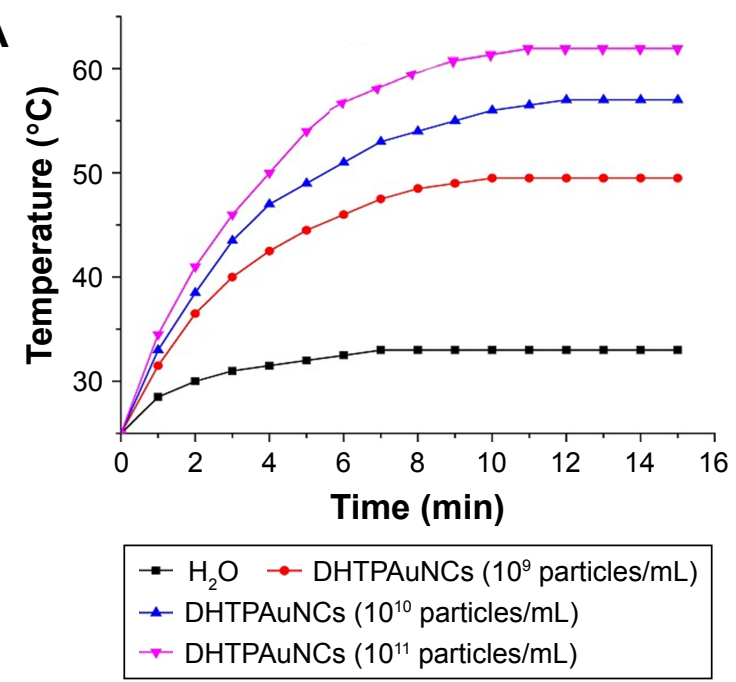

C

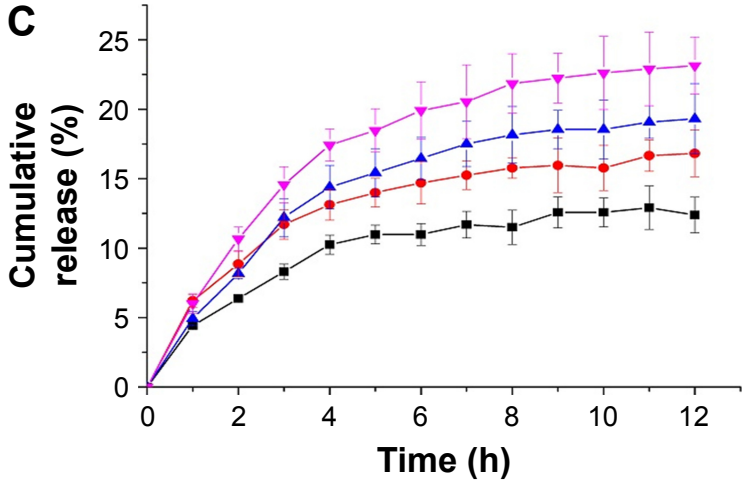

$-\mathrm{pH} 7.4 \rightarrow \mathrm{pH} 7.4+\mathrm{NIR}$
$\leftarrow \mathrm{pH} 5.5 \rightarrow \mathrm{pH} 5.5+\mathrm{NIR}$
B
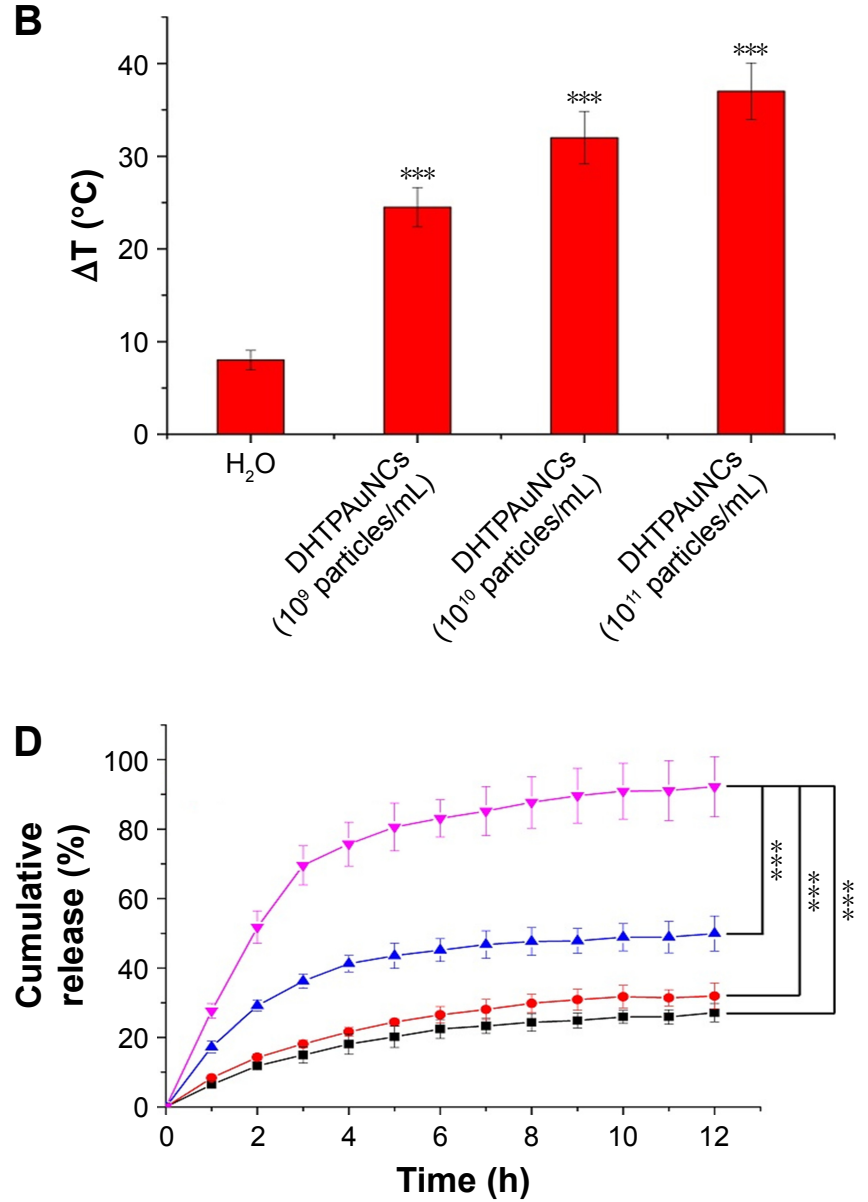

$\rightarrow \mathrm{pH} 4.5 \rightarrow \mathrm{pH} 4.5+\mathrm{NIR} \leftarrow \mathrm{pH} 4.5+$ Hyal
$\rightarrow \mathrm{pH} 4.5+\mathrm{NIR}+$ Hyal

Figure 3 Photothermal conversion and the release profile of DHTPAuNCs.

Notes: (A and B) Temperature changes of DHTPAuNC suspensions (AuNCs concentration at 109, $10^{10}$, and $10^{11}$ particles/mL) after $15 \mathrm{~min}$ of $808 \mathrm{~nm}$ laser irradiation at $1.25 \mathrm{~W} \mathrm{~cm}^{-2}(\mathrm{n}=3)$, $* * * P<0.005$, significant difference in comparison with the ultrapure water group. (C) Release profiles of DOX from DHTPAuNCs in acetate buffer $(\mathrm{pH} 5.5)$ and in the phosphate-buffered saline buffer $(\mathrm{pH} 7.4)$ with or without NIR laser irradiation. (D) Release profiles of DOX from DHTPAuNCs in pH 4.5 acetate buffer

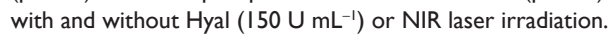

Abbreviations: AuNCs, gold nanocages; DHTPAuNCs, DOX-loaded, HA-grafted, and A54 peptide-targeted PEGylated AuNCs; DOX, doxorubicin; Hyal, hyaluronidase; NIR, near-infrared irradiation. 
could facilitate the release of encapsulated drug. The amount of encapsulated DOX in the DHTPAuNCs was quantitated to be $1.0 \mathrm{mg} \mathrm{DOX} / 16.3 \mathrm{mg}$ AuNCs as previously calculated. As shown in Figure 3C, within 12 h, in PBS buffer (pH 7.4), the DHTPAuNCs have almost no drug release. Even under NIR laser irradiation or in an acidic environment ( $\mathrm{pH} 5.5)$, only negligible drug release from the DHTPAuNCs was measured. These results demonstrated that DHTPAuNCs had a good encapsulation ability and were very stable in acidic tumor microenvironment ( $\mathrm{pH} 5.5$ ) and normal physiological conditions ( $\mathrm{pH} 7.4$ ) even with NIR laser irradiation. Then, we investigated whether the presence of Hyal had a significant effect on the drug release behavior at $\mathrm{pH} 4.5$ (Figure 3D). In comparison, an obvious burst release of DOX was assessed. This was attributed to the detachment and fragmentation of the HA on the surfaces of DHTPAuNCs in the presence of Hyal. On this basis, with NIR laser irradiation, the release of DOX was dramatically enhanced, which demonstrated the exceptional photothermal conversion efficacy of DHTPAuNCs. When DHTPAuNCs were irradiated with NIR laser, the temperature of the solution was elevated, and viscosity of the solution was reduced, which facilitated the release of DOX from the DHTPAuNCs. It has been demonstrated that the elevated temperature after NIR irrationality could accelerate the drug release. ${ }^{36,37}$ To sum up, only when HA was degraded by Hyal, such a result could be generated, during which the encapsulated drugs were released from DHTPAuNCs and the release of DOX could be accelerated by acidic $\mathrm{pH}$ and NIR. We speculated that this system was stable and could prevent any premature leakage after the particles were internalized, and make it possible to achieve controlled drug release, which would reduce unwanted adverse side effects and significantly improve the therapeutic efficacy in vitro and in vivo.

\section{Cellular uptake}

A54 peptide was reported as one of the most effective and specific peptides to liver cancer cells, especially the human HCC cell line BEL-7402. ${ }^{30}$ Herein, we examined the targeting ability of this system toward BEL-7402 cells. It was worth noting that gold nanoparticles could lead to a significant fluorescence quenching of DOX.$^{38}$ So, we investigated the uptake of non-drug-loaded AuNCs. The BEL-7402 cells were treated with HPAuNCs or HTPAuNCs (AuNCs concentration at $0.625 \times 10^{10}$ particles $\mathrm{mL}^{-1}$ ) for different times (1, 2, and 4 h). As illustrated in Figure 4A, the cells treated with HTPAuNCs exhibited stronger fluorescence intensity than those treated with HPAuNCs, indicating that the smart delivery system could efficiently enhance the cellular uptake of AuNCs. In addition, the amount of HTPAuNCs uptaken by cells is $2.919 \pm 0.111 \mathrm{ng} / 10^{6}$ cells after incubation for $10 \mathrm{~h}$, which is 1.5 -fold higher than that of the HPAuNCs
A $1 \mathrm{~h}$
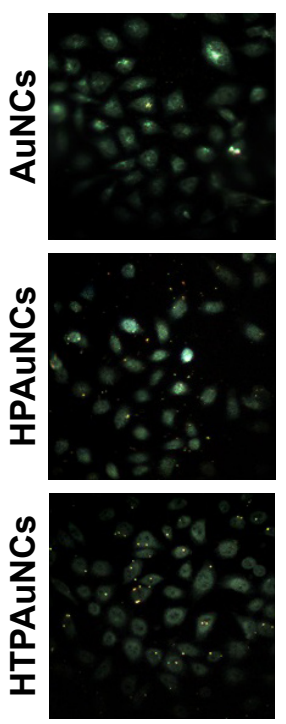

$2 \mathrm{~h}$

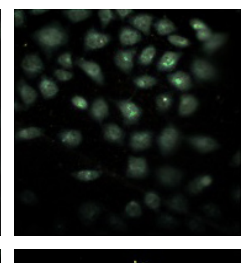

$4 \mathrm{~h}$

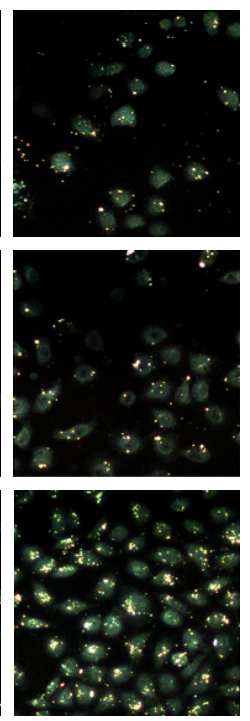

B

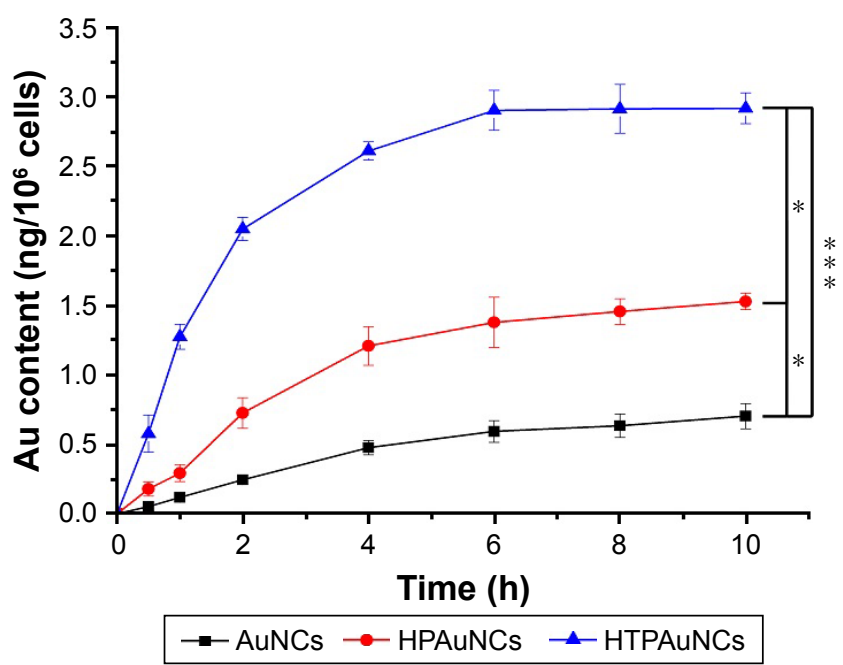

Figure 4 Cellular uptake of AuNCs, HPAuNCs, and HTPAuNCs in BEL-7402 cells.

Notes: (A) Fluorescence microscopy images of BEL-7402 cells incubated with AuNCs, HPAuNCs, or HTPAuNCs for different times (I, 2, and 4 h) (400×). (B) The amount of gold per $10^{6}$ cells ( $\mathrm{ng} / 10^{6}$ cells) determined by cell counting and inductively coupled plasma mass spectrometry, reflecting the cellular uptake. The cells were treated with HPAuNCs or HTPAuNCs for different times $(0.5,1,2,4,6$, 8, and $10 \mathrm{~h})$. Each bar was presented as the mean \pm SD $(n=3)$. $* P<0.05$, significant difference between AuNCs group and HPAuNCs group, HPAuNCs group and HTPAuNCs group. $* * * P<0.005$, significant difference between AuNCs group and HTPAuNCs group.

Abbreviations: AuNCs, gold nanocages; HPAuNCs, HA-grafted PEGylated AuNCs; HTPAuNCs, HA-grafted and A54 peptide-targeted PEGylated AuNCs; SD, standard deviation. 
( $1.528 \pm 0.059 \mathrm{ng} / 10^{6}$ cells) (Figure 4B). This result confirmed that the HTPAuNCs were more internalized to BEL-7402 cells than HPAuNCs. The specific endocytosis mediated by A54 peptide enhanced cellular uptake of HTPAuNCs.

\section{Cell viability assessment}

The targeting ability of cancer cells and the excellent photothermal conversion efficiency of this system prompt our interest to explore its therapeutic efficacy in vitro. MTT assay was performed to detect the inhibition rate of cancer cells. First, the cytotoxicity of the vehicles at various concentrations of AuNCs was assayed. In this experiment, unmodified AuNCs, HAuNCs, and HTPAuNCs are called vehicles. In BEL-7402 and L02 cells, AuNCs did not have significant toxicity (data were not shown). Next, we quantified the anticancer effect of the combined PTT and chemotherapy against BEL-7402 cells at various concentrations of DOX. The cells were treated with free DOX, DHAuNCs, HTPAuNCs, and DHTPAuNCs at various concentrations (AuNCs concentration at $0.625,1.25,2.5$, and $5.0 \times 10^{10}$ particles $\mathrm{mL}^{-1}, 1 \mathrm{mg}$ DOX/16.3 mg AuNCs). In groups without NIR irradiation, the cell inhibition rate of DHTPAuNCs was $48.18 \% \pm 2.16 \%$, higher than that of DHPAuNCs $(39.68 \% \pm 1.86 \%)$ and similar to that of free DOX $(52.01 \% \pm 1.13 \%)$ with the concentration of AuNCs at $2.5 \times 10^{10}$ particles $\mathrm{mL}^{-1}$ (Figure 5A). This result further confirmed the targeting ability. As illustrated in Figure 5B, the cancer cells irradiated by NIR without the presence of AuNCs exhibited a high cell viability compared with the control group, which demonstrated that only NIR laser irradiation did not affect the growth of the cancer cells. However, the cells cultured with HTPAuNCs (AuNCs at $2.5 \times 10^{10}$ particles $\mathrm{mL}^{-1}$ ) upon NIR laser irradiation showed a cell mortality rate of $30.49 \% \pm 2.91 \%$, which was attributed to the localized heat generated by HTPAuNCs. Meanwhile,
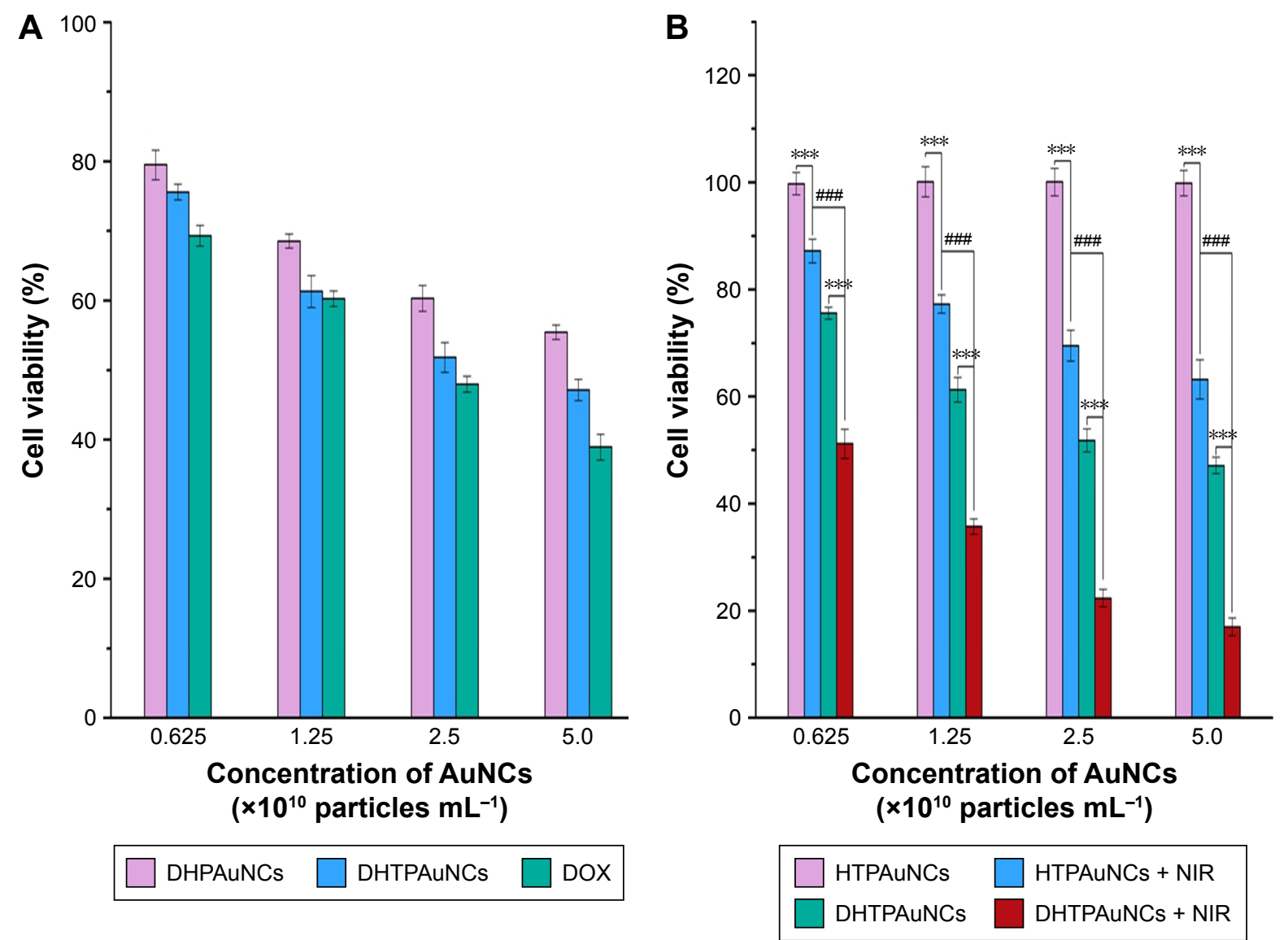

Figure 5 Cytotoxicity of BEL-7402 cells.

Notes: (A) The cell viability of BEL-7402 cells treated with DHPAuNCs, DHTPAuNCs, and DOX (AuNCs at the concentration of $0.625 \times 10^{10}$ particles/mL, I.25 $\times 10^{10}$ particles $/ \mathrm{mL}, 2.5 \times 10^{10}$ particles $/ \mathrm{mL}$, and $5.0 \times 10^{10}$ particles $/ \mathrm{mL}$, respectively, and the weight ratio of DOX/AuNCs was I/I6.3) for $24 \mathrm{~h}$. (B) Cell viability of BEL-7402 cells exposed to phosphate-buffered saline, HTPAuNCs, and DHTPAuNCs for $24 \mathrm{~h}$. After $6 \mathrm{~h}$ incubation, NIR laser $\left(808 \mathrm{~nm}, 1.25 \mathrm{~W} \mathrm{~cm}^{-2}\right.$, $\left.10 \mathrm{~min}\right)$ was used to achieve photothermal therapy. Each bar was presented as the mean $\pm S D(n=4)$. $* * * P<0.005$, significant difference between irradiated groups and unirradiated groups. $P\left({ }^{\prime}+0.005\right.$, significant difference between HTPAuNCs + NIR group and DHTPAuNCs + NIR group.

Abbreviations: AuNCs, gold nanocages; DHTPAuNCs, DOX-loaded, HA-grafted, and A54 peptide-targeted PEGylated AuNCs; DOX, doxorubicin; NIR, near-infrared irradiation; HTPAuNCs, HA-grafted and A54 peptide-targeted PEGylated AuNCs; SD, standard deviation. 
when the cells were incubated with DHTPAuNCs (AuNCs at $2.5 \times 10^{10}$ particles $\mathrm{mL}^{-1}$ ) upon NIR laser irradiation, the cell inhibition rate significantly increased to $77.63 \% \pm 1.63 \%$, much higher than that of the sole PTT and single chemotherapy (Figure 5B). The experiment results proved that more drugs could be delivered into tumor cells by DHTPAuNCs, and this novel delivery system could show synergistic effect of photothermal-chemotherapy in vitro.

\section{Antitumor effect in vivo}

To explore whether DHTPAuNCs could significantly improve the antitumor effect in vivo, the tumor growth inhibition ability of the AuNCs-based nanocomposites with or without NIR laser irradiation was compared in BEL-7402 tumor-bearing nude mice $\left(\sim 150 \mathrm{~mm}^{3}\right)$. Meanwhile, free DOX was used as positive control and NS was applied as negative control. An adequate concentration of the nanocomposites localized within tumor, which is treated as exogenous energy absorbers that convert laser energy into heat, is a prerequisite for the following PTT. First, the photothermal conversion efficiency of the AuNCs-based nanocomposites in vivo was evaluated by the temperature changes of the tumor site. Localized heating was observed in the tumor regions of the mice, which were treated with the AuNCs-based nanocomposites and NIR irradiation. Temperature in the tumor region increased rapidly from about $36.2^{\circ} \mathrm{C}$ (Figure $6 \mathrm{~A}-\mathrm{a}-\mathrm{d}$ ) to $40.0^{\circ} \mathrm{C}$ (AuNCs group, Figure $6 \mathrm{~A}-\mathrm{f}$ ), $45.0^{\circ} \mathrm{C}$ (HPAuNCs group, Figure $6 \mathrm{~A}-\mathrm{g}$ ), and $49.0^{\circ} \mathrm{C}$ (HTPAuNCs group, Figure 6A-h) because of the exceptional photothermal conversion efficacy of AuNCs selectively accumulated in the tumor region. However, the temperature in the tumor site of NS group still remained under $40.0^{\circ} \mathrm{C}$ after NIR laser irradiation for $10 \mathrm{~min}$ (Figure 6A-e). It has been reported that cancer cells, with lower heat tolerance, could be killed by a high temperature over $43{ }^{\circ} \mathrm{C} .{ }^{39}$ Hence, DHTPAuNCs could be applied as photothermal agents for cancer therapy. In addition, the differences in the elevated temperature in the tumor regions of AuNCs group, HPAuNCs group, and HTPAuNCs group suggested the targeting ability. Subsequently, the changes in relative tumor volume $\left(\mathrm{RTV}, \mathrm{V} / \mathrm{V}_{0}\right)$ were applied to assess the antitumor effect of DHTPAuNCs-mediated photothermal-chemotherapy in vivo (Figure 6C). After five treatments, the control groups (NS, NS + NIR group, and HTPAuNCs group) resulted in RTV of $368 \% \pm 22 \%$, $365 \% \pm 45 \%$, and $359 \% \pm 29 \%$, respectively. DHTPAuNCs group showed RTV of $214 \% \pm 39 \%$, slightly higher than that

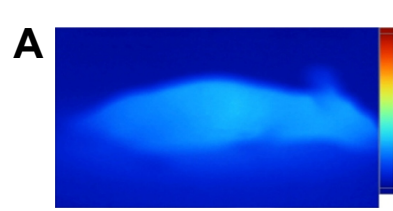

a

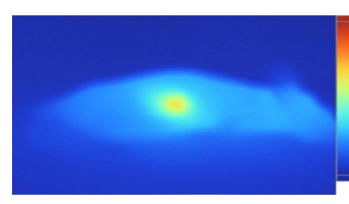

e
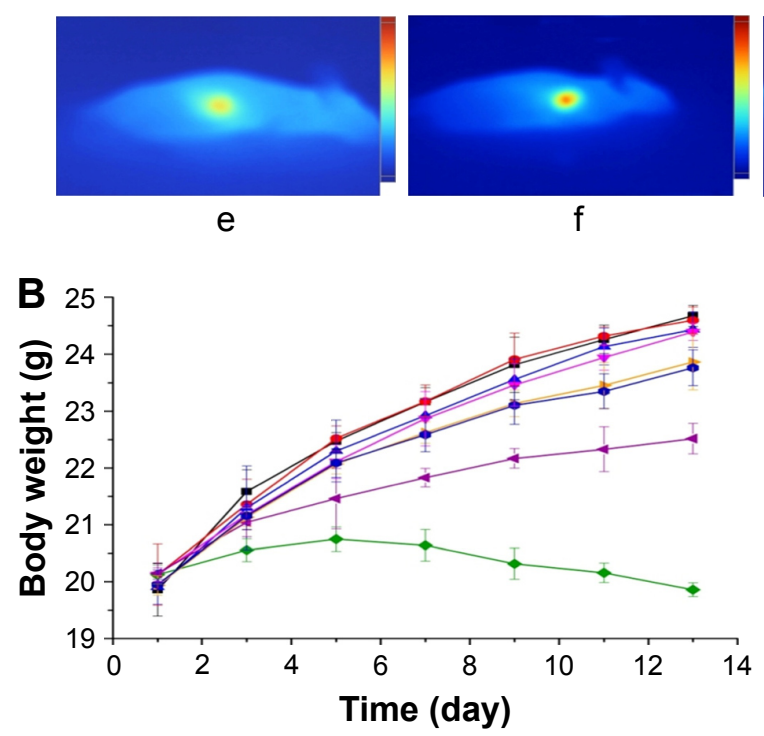

f

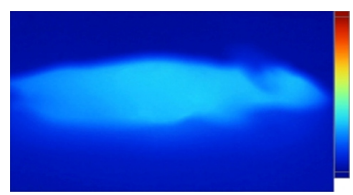

$\rightarrow$ NS $\rightarrow$ NS + NIR $\leftarrow$ HTPAuNCs $\rightarrow$ HTPAuNCs + NIR
$\rightarrow$ DOX $\leftarrow$ DHPAuNCs $\rightarrow$ DHTPAuNCs $\rightarrow$ DHTPAuNCs + NIR

C
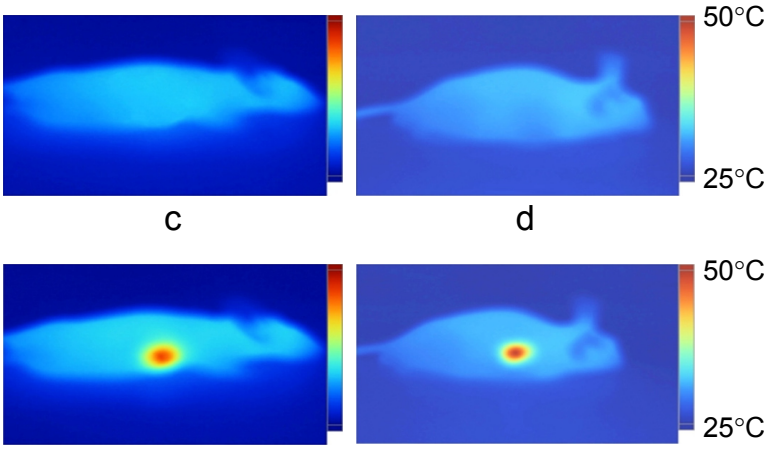

g

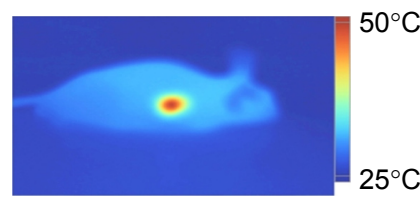

h

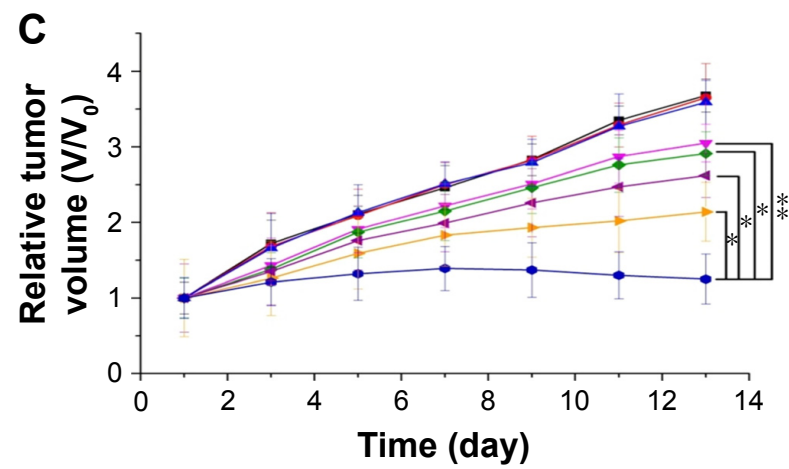

Figure 6 (Continued) 


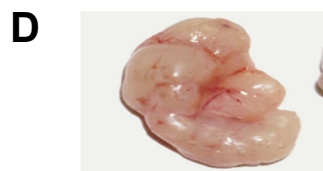

a

E

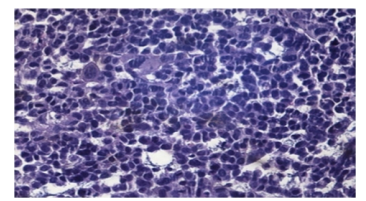

a

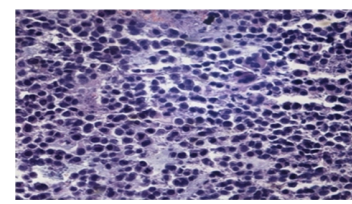

e

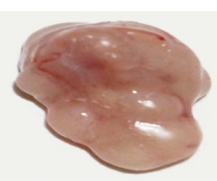

b

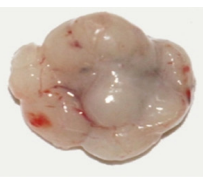

C

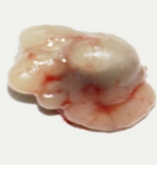

d

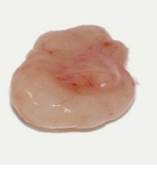

e

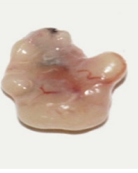

$f$

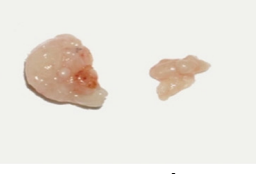

g h

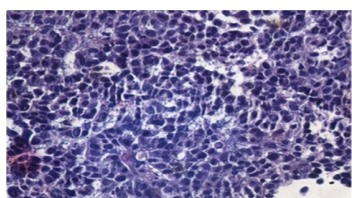

b

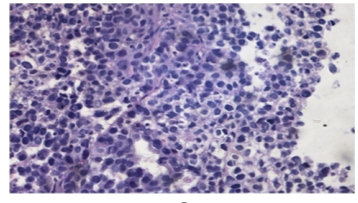

$\mathrm{f}$

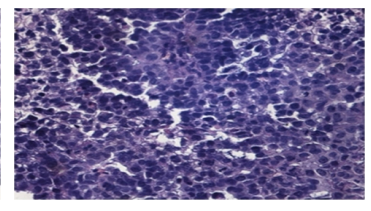

C

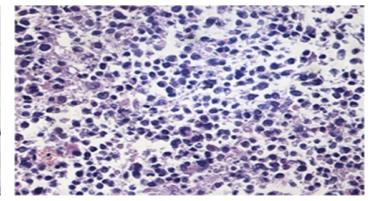

g

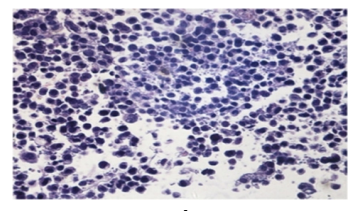

d

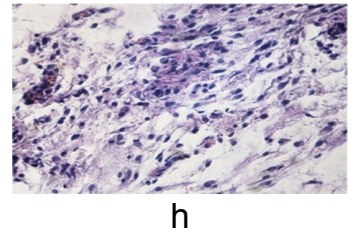

Figure 6 Antitumor efficacy under NIR irradiation in vivo, and infrared thermal imaging.

Notes: (A) Infrared thermal images of the mice before (a-d) or after $10 \mathrm{~min}(\mathrm{e}-\mathrm{h})$ of $808 \mathrm{~nm}$ NIR laser irradiation at $1.25 \mathrm{~W} \mathrm{~cm}^{-2}$ and the color bar related the relative temperature values. The mice were injected systemically with NS (a, e), AuNCs (b, f), HPAuNCs (c, g), or HTPAuNCs (d, h). (B) Changes in the body weight of the mice with the time. (C) The tumor growth curves of eight groups. (D) Representative images of the tumors harvested from the mice after treatments. (E) Histomorphological assessments of tumor sections stained with hematoxylin-eosin after the treatment for 15 days. (B), (C), (D), and (E) grouping were as follows: (a) NS group; (b) NS + NIR group; (c) HTPAuNCs group; (d) HTPAuNCs + NIR group; (e) free DOX group; (f) DHPAuNCs group; (g) DHTPAuNCs group; (h) DHTPAuNCs + NIR group.

Abbreviations: AuNCs, gold nanocages; DHTPAuNCs, DOX-loaded, HA-grafted, and A54 peptide-targeted PEGylated AuNCs; DOX, doxorubicin; HPAuNCs, HA-grafted PEGylated AuNCs; HTPAuNCs, HA-grafted and A54 peptide-targeted PEGylated AuNCs; NIR, near-infrared irradiation; NS, normal saline.

of free DOX $(291 \% \pm 29 \%)$ because of its targeting ability. The group of DHPAuNCs with the EPR effect also showed similar RTV of $262 \% \pm 29 \%$. Meanwhile, HTPAuNCs + NIR group achieved RTV of $305 \% \pm 25 \%$, suggesting that the single chemotherapy or the sole PTT could not achieve desirable inhibition of tumor growth. The mice treated with DHTPAuNCs + NIR showed RTV of $125 \% \pm 33 \%$, because of its synergistic manner. So, antitumor effect in vivo could be dramatically improved by the photothermal-chemotherapy, which was consistent with the results obtained in vitro. In all groups, we did not observe obvious loss of body weights. This result further proved that functionalized AuNCs had good biocompatibility (Figure 6B). Representative images of the tumors harvested from the mice after treatments shown in Figure 6D confirmed the enhanced therapeutic effect mediated by synergistic therapy. In addition, we performed a histopathological examination in tumors. As illustrated in Figure 6E, the morphology form of tumor tissue of NS + NIR group and HTPAuNCs group was mainly similar to that of NS group, and all of them had no obvious damage, which indicated that it would not cause cellular damage only with NIR laser irradiation, and the non-drug-loaded carrier was almost nontoxic to cells. Compared with the DOX group, both DHPAuNCs and DHTPAuNCs groups caused more tumor cell apoptosis. The reason was that the carrier of nanomaterials could target passively to the tumor site, and increased the accumulation of the drug in the tumor tissue. Most cell death has been observed in the DHTPAuNCs + NIR group among all other groups, which indicated that the chemo-photothermal therapy exerted a better antitumor effect than the single chemotherapy and the sole photothermal therapy, respectively.

\section{Serum biochemical analysis}

To further test the toxicity of the nanocomposites, we evaluated kidney function and liver function with serum levels of CREA, UREA, TNF- $\alpha$, AST, and ALT (Table 1). When liver cells are destroyed or necrosis happens, intracellular enzymes can be released into the blood, so the detection of the level of liver enzyme in the blood is one of the most common methods in research of liver toxicity. In this experiment, we chose ALT and AST, which are the most representative indicators to understand the impact of carrier materials on liver. ${ }^{40}$ A large number of toxins entered the liver and the product of the metabolism of liver cells can be transported to the kidney through a long circulation system, and among them, $80 \%$ of renal blood flow will get to the renal cortex. TNF- $\alpha$ is a kind of important cytokines produced by activated mononuclear macrophages in the process of acute liver necrosis, which is the early reaction of the liver damage. A large number of clinical and experimental researches showed that the liver of patients with liver damage produced a lot of TNF- $\alpha$, and 
Table I The influence of HTPAuNCs on liver function and kidney function after treatment

\begin{tabular}{lllllll}
\hline Group & Time $(\mathbf{h})$ & ALT (U/L) & AST (U/L) & $\begin{array}{l}\text { CREA } \\
(\mu \mathrm{mol} / \mathrm{L})\end{array}$ & $\begin{array}{l}\text { UREA } \\
(\mathbf{m m o l} / \mathbf{L})\end{array}$ & $\begin{array}{l}\text { TNF- } \alpha \\
(\mathbf{p g} / \mathbf{m L})\end{array}$ \\
\hline NS & 0 & $35.35 \pm 4.16$ & $121.56 \pm 18.84$ & $46.41 \pm 3.18$ & $7.26 \pm 1.28$ & $26.56 \pm 0.09$ \\
& 12 & $35.58 \pm 6.21$ & $126.58 \pm 29.05$ & $46.58 \pm 2.09$ & $7.58 \pm 2.19$ & $26.58 \pm 0.09$ \\
& 24 & $36.63 \pm 5.10$ & $136.63 \pm 18.72$ & $45.63 \pm 3.87$ & $7.63 \pm 1.87$ & $26.63 \pm 0.09$ \\
& 72 & $37.85 \pm 6.03$ & $116.45 \pm 21.12$ & $47.45 \pm 4.11$ & $7.25 \pm 2.28$ & $26.45 \pm 0.11$ \\
& 120 & $35.52 \pm 6.22$ & $126.52 \pm 13.21$ & $46.51 \pm 3.32$ & $7.51 \pm 1.32$ & $26.52 \pm 0.13$ \\
HTPAuNCs & 0 & $35.65 \pm 5.21$ & $126.59 \pm 18.52$ & $46.59 \pm 1.85$ & $7.69 \pm 1.68$ & $26.59 \pm 0.09$ \\
& 12 & $36.50 \pm 7.35$ & $136.74 \pm 27.57$ & $45.15 \pm 2.71$ & $7.49 \pm 1.56$ & $26.93 \pm 0.07$ \\
& 24 & $34.24 \pm 5.72$ & $133.43 \pm 17.38$ & $47.97 \pm 4.79$ & $6.97 \pm 1.72$ & $26.88 \pm 0.11$ \\
& 72 & $37.22 \pm 6.53$ & $119.72 \pm 20.14$ & $47.62 \pm 5.23$ & $7.53 \pm 1.67$ & $26.53 \pm 0.13$ \\
& 120 & $35.63 \pm 8.74$ & $125.71 \pm 11.62$ & $48.59 \pm 3.27$ & $7.74 \pm 1.09$ & $26.39 \pm 0.10$ \\
\hline
\end{tabular}

Notes: The serum levels of ALT and AST were assessed to index the liver function. The serum levels of CREA and UREA were evaluated to indicate the kidney function. All data were presented as the mean \pm SD $(n=5)$.

Abbreviations: ALT, alanine aminotransferase; AST, aspartate aminotransferase; CREA, creatinine; HTPAuNCs, HA-grafted, and A54 peptide-targeted PEGylated AuNCs; SD, standard deviation; TNF- $\alpha$, tumor necrosis factor- $\alpha$; UREA, urea.

the occurrence and development of hepatitis, liver cirrhosis, and liver cancer were closely related with the TNF- $\alpha{ }^{41}$ Animal experimental results demonstrated that the content of TNF- $\alpha$ of the HTPAuNCs group had no significant increase compared with the NS group (Table 1). In addition, the dysfunction of the kidney will make the concentration of renal tubular solution increased and the renal tubular epithelial cells damaged. So the contents of UREA and CREA are the most common and economic indicator used to detect kidney injury. ${ }^{41,42}$ Animal experimental results showed that our drug carrier HTPAuNCs did not cause obvious inflammatory reaction, liver toxicity, and kidney toxicity within the treatment period (Table 1), which were consistent with the experimental results in vitro.

\section{Conclusion}

In summary, we have successfully developed a liver cancerspecific targeting drug delivery system based on DOXloaded, A54 peptide-targeted, HA-grafted PEGylated AuNCs (DHTPAuNCs) for cancer therapy. Our results showed that the intracellular enzyme Hyal could trigger DOX release from DHTPAuNC, while NIR laser and acidic $\mathrm{pH}$ could accelerate this process. We evaluated DHTPAuNCs-mediated chemophotothermal therapeutic effect both in vitro and in vivo. The cellular inhibition rate of integrated therapy mediated by the DHTPAuNCs was the highest among all of the groups $(77.63 \% \pm 1.63 \%)$. Synergistic treatment of chemotherapy and PTT remarkably delayed tumor growth by RTV of $125 \% \pm 33 \%$. The serum biochemical analysis demonstrated good bio-compliance of the DHTPAuNCs in vivo. Therefore, this system has great potential for application as a delivery system for enhanced therapeutic efficiency with limited toxicity for HCC treatment.

\section{Acknowledgments}

This work was supported by NSFC under award number 81272563 and Natural Science Foundation of Henan Province under award number 162300410044.

\section{Disclosure}

The authors report no conflicts of interest in this work.

\section{References}

1. Ferlay J, Soerjomataram I, Dikshit R, et al. Cancer incidence and mortality worldwide: sources, methods and major patterns in GLOBOCAN 2012. Int J Cancer. 2015;136(5):E359-E386.

2. Dhanasekaran R, Venkatesh SK, Torbenson MS, Roberts LR. Clinical implications of basic research in hepatocellular carcinoma. J Hepatol. 2016;64(3):736-745.

3. El-Serag HB. Hepatocellular carcinoma. N Engl J Med. 2011;365(12): 1118-1127.

4. Chen MC, Lin ZW, Ling MH. Near-infrared light-activatable microneedle system for treating superficial tumors by combination of chemotherapy and photothermal therapy. ACS Nano. 2016;10(1):93-101.

5. Kim J, Kim J, Jeong C, Kim WJ. Synergistic nanomedicine by combined gene and photothermal therapy. Adv Drug Deliv Rev. 2016;98:99-112.

6. He X, Bao X, Cao H, et al. Tumor-penetrating nanotherapeutics loading a near-infrared probe inhibit growth and metastasis of breast cancer. Adv Funct Mater. 2015;25(19):2831-2839.

7. Li Z, Hu Y, Howard KA, et al. Multifunctional bismuth selenide nanocomposites for antitumor thermo-chemotherapy and imaging. ACS Nano. 2016;10(1):984-997.

8. Espinosa A, Di Corato R, Kolosnjaj-Tabi J, Flaud P, Pellegrino T, Wilhelm C. Duality of iron oxide nanoparticles in cancer therapy: amplification of heating efficiency by magnetic hyperthermia and photothermal bimodal treatment. ACS Nano. 2016;10(2):2436-2446.

9. Song J, Yang X, Jacobson O, et al. Ultrasmall Gold nanorod vesicles with enhanced tumor accumulation and fast excretion from the body for cancer therapy. Adv Mater. 2015;27(33):4910-4917.

10. Huang S, Duan S, Wang J, et al. Folic-acid-mediated functionalized gold nanocages for targeted delivery of anti-miR-181b in combination of gene therapy and photothermal therapy against hepatocellular carcinoma. Adv Funct Mater. 2016;26:2532-2544.

11. Potara M, Boca S, Licarete E, et al. Chitosan-coated triangular silver nanoparticles as a novel class of biocompatible, highly sensitive plasmonic platforms for intracellular SERS sensing and imaging. Nanoscale. 2013;5(13):6013-6022. 
12. Meng Z, Wei F, Wang R, et al. NIR-laser-switched in vivo smart nanocapsules for synergic photothermal and chemotherapy of tumors. Adv Mater. 2016;28(2):245-253.

13. Guo M, Huang J, Deng Y, et al. pH-responsive cyanine-grafted graphene oxide for fluorescence resonance energy transfer-enhanced photothermal therapy. Adv Funct Mater. 2015;25(1):59-67.

14. Marangon I, Ménard-Moyon C, Silva AKA, Bianco A, Luciani N, Gazeau F. Synergic mechanisms of photothermal and photodynamic therapies mediated by photosensitizer/carbon nanotube complexes. Carbon. 2016;97:110-123.

15. Song XR, Wang X, Yu SX, et al. $\operatorname{Co}(9) \operatorname{Se}(8)$ nanoplates as a new theranostic platform for photoacoustic/magnetic resonance dual-modalimaging-guided chemo-photothermal combination therapy. Adv Mater. 2015;27(21):3285-3291.

16. Lin L, Cong Z, Cao J, et al. Multifunctional $\mathrm{Fe}_{3} \mathrm{O}_{4} @$ polydopamine core-shell nanocomposites for intracellular mRNA detection and imaging-guided photothermal therapy. ACS Nano. 2014;8(4): 3876-3883.

17. Deng K, Hou Z, Deng X, Yang P, Li C, Lin J. Enhanced antitumor efficacy by $808 \mathrm{~nm}$ laser-induced synergistic photothermal and photodynamic therapy based on a indocyanine-green-attached W18049 nanostructure. Adv Funct Mater. 2015;25(47):7280-7290.

18. Sun M, Liu F, Zhu Y, et al. Salt-induced aggregation of gold nanoparticles for photoacoustic imaging and photothermal therapy of cancer. Nanoscale. 2016;8(8):4452-4457.

19. Chen J, Saeki F, Wiley BJ, et al. Gold nanocages: bioconjugation and their potential use as optical imaging contrast agents. Nano Lett. 2005;5(3):473-477.

20. Choi KY, Yoon HY, Kim J-H, et al. Smart nanocarrier based on PEGylated hyaluronic acid for cancer therapy. ACS Nano. 2011;5(11): 8591-8599.

21. Qhattal HSS, Hye T, Alali A, Liu X. Hyaluronan polymer length, grafting density, and surface poly(ethylene glycol) coating influence in vivo circulation and tumor targeting of hyaluronan-grafted liposomes. ACS Nano. 2014;8(6):5423-5440.

22. Chen Z, Li Z, Wang J, et al. A multi-synergistic platform for sequential irradiation-activated high-performance apoptotic cancer therapy. $A d v$ Funct Mater. 2014;24(4):522-529.

23. Harada H, Takahashi M. CD44-dependent intracellular and extracellular catabolism of hyaluronic acid by hyaluronidase-1 and -2. J Biol Chem. 2007;282(8):5597-5607.

24. Aruffo A, Stamenkovic I, Melnick M, Underhill CB, Seed B. CD44 is the principal cell surface receptor for hyaluronate. Cell. 1990;61(7): 1303-1313

25. David N, Ronit S, Dvorah I-S. CD44: structure, function, and association with the malignant process. Adv Cancer Res. 1997;71:241-319.

26. Toole BP. Hyaluronan-CD44 interactions in cancer: paradoxes and possibilities. Clin Cancer Res. 2009;15(24):7462-7468.
27. Zhou B, Weigel JA, Saxena A, Weigel PH. Molecular cloning and functional expression of the rat $175-\mathrm{kDa}$ hyaluronan receptor for endocytosis. Mol Biol Cell. 2002;13(8):2853-2868.

28. Ngoune R, Peters A, von Elverfeldt D, Winkler K, Putz G. Accumulating nanoparticles by EPR: a route of no return. J Controll Release. 2016;238:58-70.

29. Liu X, Braun GB, Zhong H, et al. Tumor-targeted multimodal optical imaging with versatile cadmium-free quantum dots. Adv Funct Mater. 2016;26(2):267-276.

30. Du B, Han H, Wang Z, et al. Targeted drug delivery to hepatocarcinoma in vivo by phage-displayed specific binding peptide. Mol Cancer Res. 2010;8(2):135-144.

31. Skrabalak SE, Au L, Li X, Xia Y. Facile synthesis of Ag nanocubes and Au nanocages. Nat Protoc. 2007;2(9):2182-2190.

32. Wang Y, Black KCL, Luehmann H, et al. Comparison study of gold nanohexapods, nanorods, and nanocages for photothermal cancer treatment. ACS Nano. 2013;7(3):2068-2077.

33. Jahn B, Martin E, Stueben A, Bhakdi S. Susceptibility testing of Candida albicans and Aspergillus species by a simple microtiter menadioneaugmented 3-(4,5-dimethyl-2-thiazolyl)-2,5-diphenyl-2H-tetrazolium bromide assay. J Clin Microbiol. 1995;33(3):661-667.

34. Fella C, Walker GF, Ogris M, Wagner E. Amine-reactive pyridylhydrazone-based $\mathrm{PEG}$ reagents for $\mathrm{pH}$-reversible $\mathrm{PEI}$ polyplex shielding. Eur J Pharm Sci. 2008;34(4-5):309-320.

35. Yi DK, Sun IC, Ryu JH, et al. Matrix metalloproteinase sensitive gold nanorod for simultaneous bioimaging and photothermal therapy of cancer. Bioconjug Chem. 2010;21(12):2173-2177.

36. Li DD, Wang JX, Ma Y, et al. A donor-acceptor conjugated polymer with alternating isoindigo derivative and bithiophene units for nearinfrared modulated cancer thermo-chemotherapy. ACS Appl Mater Interfaces. 2016;8(30):19312-19320.

37. Wang J, Liu Y, Ma Y, et al. NIR-activated supersensitive drug release using nanoparticles with a flow core. Adv Funct Mater. 2016; 26(41):7516-7525.

38. Wang F, Wang YC, Dou S, Xiong MH, Sun TM, Wang J. Doxorubicintethered responsive gold nanoparticles facilitate intracellular drug delivery for overcoming multidrug resistance in cancer cells. ACS Nano. 2011;5(5):3679-3692.

39. Kaplowitz N. Drug-induced liver injury. Clin Infect Dis. 2004;38(Supp12): S44-S48

40. Schwabe RF, Brenner DA. Mechanisms of liver injury. I. TNF- $\alpha-$ induced liver injury: role of IKK, JNK, and ROS pathways. Am J Physiol Gastrointest Liver Physiol. 2006;290(4):G583-G589.

41. Dzhekovastojkova S, Bogdanska J, Stojkova Z. Peroxisome proliferators: their biological and toxicological effects. Clin Chem Lab Med. 2001; 39(6):468-474.

42. Zhang X, Wu D, Shen X, et al. Size-dependent in vivo toxicity of PEGcoated gold nanoparticles. Int J Nanomed. 2011;6:2071-2081.
International Journal of Nanomedicine

\section{Publish your work in this journal}

The International Journal of Nanomedicine is an international, peerreviewed journal focusing on the application of nanotechnology in diagnostics, therapeutics, and drug delivery systems throughout the biomedical field. This journal is indexed on PubMed Central, MedLine, CAS, SciSearch ${ }^{\circledR}$, Current Contents ${ }^{\circledR} /$ Clinical Medicine,

\section{Dovepress}

Journal Citation Reports/Science Edition, EMBase, Scopus and the Elsevier Bibliographic databases. The manuscript management system is completely online and includes a very quick and fair peer-review system, which is all easy to use. Visit http://www.dovepress.com/ testimonials.php to read real quotes from published authors. 